\title{
Functional mechanism of the enhancement of 5-fluorouracil sensitivity by TUSC4 in colon cancer cells
}

\author{
MING-NA LIU, AI-YUN LIU, FENG-HUA PEI, XIAO MA, YU-JING FAN, YA-JU DU and BING-RONG LIU \\ Department of Gastroenterology, The Second Affiliated Hospital of Harbin Medical University, \\ Harbin, Heilongjiang 150080, P.R. China
}

Received October 11, 2014; Accepted July 7, 2015

DOI: 10.3892/ol.2015.3801

\begin{abstract}
Fluorouracil (5-FU) is the chemotherapeutic drug of choice for the treatment of metastatic colorectal cancer (CRC). Tumor suppressor candidate 4 (TUSC4), also referred to as nitrogen permease regulator-like 2 (NPRL2), is located at chromosome 3p21.3 and expressed in numerous normal tissues, including the heart, liver, skeletal muscle, kidney, and pancreas. The aim of the present study was to investigate the functional mechanism by which TUSC4 affects sensitivity to 5-FU and to determine its clinical significance in CRC. The results of the present study demonstrated that TUSC4 overexpression increases the sensitivity of HCT116 cells to 5-FU. The $\mathrm{IC}_{50}$ of 5-FU was reduced in cells transduced with TUSC4 compared with negative control (NC) cells, and the effect of TUSC4 on 5-FU sensitivity was time dependent. Following TUSC 4 transduction in HCT116 cells, a proportion of the cells were arrested in the G1 phase of the cell cycle, and a reduction in the $S$ phase population was observed. Flow cytometry analysis revealed that TUSC4 transduction and 5-FU treatment increased apoptosis compared with NC cells. The mechanism through which TUSC4 overexpression enhances 5-FU sensitivity involves the downregulation of the function of the PI3K/Akt/mTOR network. Furthermore, 5-FU upregulated caspase-3 and caspase-9, promoting apoptosis in TUSC4-overexpressing cells compared with cells that were transduced with TUSC4 or treated with 5-FU and NC cells. The findings of the present study indicate that TUSC4 has potential as a biomarker for the prediction of the response to 5-FU and prognosis in patients with colorectal cancer and other types of human cancer. TUSC4 may also act as a molecular therapeutic agent for enhancing the patient's response to 5-FU treatment.
\end{abstract}

Correspondence to: Dr Bing-Rong Liu, Department of Gastroenterology, The Second Affiliated Hospital of Harbin Medical University, 148 Baojian Road, Harbin, Heilongjiang 150080, P.R. China

E-mail: liubingrong@medmail.com.cn

Key words: TUSC4, colorectal cancer, PI3K/Akt/mTOR, 5-fluorouracil

\section{Introduction}

Colorectal cancer (CRC) is the second leading cause of adult cancer-associated mortality in the USA and it is associated with a low survival rate (1). Chemotherapeutic compounds that are currently used to treat colorectal cancer include 5-flurouracil (5-FU), oxaliplatin, and irinotecan (2). 5-FU is routinely used for the management of patients with CRC (3). Treating cells in vitro with 5-FU results in DNA damage, specifically double-strand (and single-strand) breaks occur during $\mathrm{S}$ phase due to the misincorporation of the metabolite of 5-FU, FdUTP, into the DNA of the cell (4). However, the use of 5-FU as a colorectal cancer chemotherapeutic agent has been somewhat limited due to the toxicity, limited success and adverse side effects associated with 5-FU treatment. As such identifying and developing novel and safe treatment strategies that may enhance the tumor cell response and overcome chemoresistance to antitumor drugs.

The tumor suppressor candidate 4 (TUSC4), also referred to as nitrogen permease regulator like 2 (NPRL2), is one of the candidate tumor suppressor genes identified in human chromosome 3 p21.3 region in which genomic abnormalities, including a loss of heterozygosity and homozygous deletion, are frequently observed in the early stages of the development of various types of human cancer (5-7). The overexpression of TUSC4 inhibits proliferation and induces apoptosis in a variety of tumor cell lines (8). Previous studies have demonstrated that TUSC4 induces susceptibility to anticancer drugs and apoptosis $(9,10)$. Additional studies have indicated that TUSC4 is involved in DNA mismatch repair, cell cycle checkpoint signaling, and the regulation of apoptosis $(5,11)$.

Previous studies have reported that TUSC4 is a potential biomarker for predicting a patient's response to cisplatin in addition to the prognosis of patients with lung and other types of cancer; TUSC4 is also a molecular therapeutic agent for enhancing and resensitizing the response of nonresponders to cisplatin treatment $(10,12)$. However, how TUSC4 suppresses tumor proliferation and whether TUSC4 affects the sensitivity of CRC cells to chemotherapy remains unknown. In the present study, the colorectal cancer cell line HCT116 was used to determine the effects of the TUSC4 signaling pathway on apoptosis induced by the chemotherapeutic drug 5-FU to further elucidate the role of the TUSC4 signaling pathway in increasing the 5-FU sensitivity in these cells to 
contribute to the identification of an effective treatment for CRC.

\section{Materials and methods}

Cell culture. The colon cancer cell line HCT116 was purchased from the Chinese Academy of Sciences (Shanghai, China). The cells were cultured in RPMI-1640 medium supplemented with $10 \%$ fetal bovine serum (HyClone, Logan, UT, USA) and $1 \%$ penicillin/streptomycin (Beyotime Institute of Biotechnology, Haimen, China) in a humidified atmosphere of $5 \% \mathrm{CO}_{2}$ at $37^{\circ} \mathrm{C}$. Cells were passaged every 2-3 days through digestion with $0.25 \%$ trypsin. Logarithmically growing cells were prepared.

Transductions and assay. The full length human TUSC4 (NPRL2) gene (GenBankaccession no. NM_006545) was purchased from Shanghai Genechem Co. Ltd. (Shanghai, China) as a fusion with enhanced green fluorescence protein (eGFP) in the GV208 vector. The lentiviral vector system consisted of GV208 and the pHelper 1.0 and pHelper 2.0 packaging vectors. The three vectors were cotransfected into 293T cells in serum-free medium using Lipofectamine 2000 (Invitrogen Life Technologies, Carlsbad, CA, USA). The medium was changed to complete medium after $8 \mathrm{~h}$ of incubation. High-titer recombinant lentiviruses encoding TUSC4 were harvested $48 \mathrm{~h}$ after transfection. HCT116 cells in the log phase were seeded at $5 \times 10^{5}$ cells/well in 96 -well plates and transduced with TUSC4-GFP or GFP lentiviruses in serum-free medium. Polybrene was added to improve the transduction efficiency. After $8 \mathrm{~h}$, the medium was changed to complete medium. At $72 \mathrm{~h}$ after transduction, GFP expression was examined by fluorescence microscopy (TE2000; Nikon Corporation, Toyko, Japan) and a luciferase assay was performed in HCT116 cells. The protein expression levels were analyzed $72 \mathrm{~h}$ after transduction. All experiments were performed in triplicate, and the representative results are reported.

Cell viability assay. Non-transduced and transduced cells were dispersed and seeded at $5 \times 10^{3}$ cells/well in 96-well microplates. After $24 \mathrm{~h}$, freshly prepared 5-FU (Jinyao Amino Acid Co., Ltd., Tianjin, China) was used to determine the optimal concentration and time course of the HCT116 cell response to 5-FU. Cell viability was assessed with the cell counting kit-8 (CCK-8, Dojindo Molecular Technologies, Inc., Kumamoto, Japan) assay following application of various concentrations of 5-FU $(1.25,2.5,5,10$ and $20 \mu \mathrm{M})$ and incubating for a variety of time points $(24,48$ and $72 \mathrm{~h})$ in culture. The absorbance value (A) at $450 \mathrm{~nm}$ was read using a microplate reader (Thermo Fisher Scientific, Inc., Rockford, IL, USA). At least 3 independent experiments were performed in quadruplicate.

Flow cytometry (FCM) analysis of the cell cycle and apoptosis. Cells transduced with TUSC4 were treated with $5 \mu \mathrm{M}$ of 5-FU for $48 \mathrm{~h}$ and harvested. After trypsinization, the cells were washed with phosphate-buffered saline (PBS) and subsequently fixed in $85 \%$ ethanol. Following fixation, the cells were washed with PBS/1\% fetal calf serum (FCS), resuspended in PBS $/ 1 \%$ FCS containing $5 \mu \mathrm{M}$ PI and $250 \mu \mathrm{g} / \mathrm{ml}$ RNase A
(Multisciences Biotech, Hangzhou, China), and then incubated for $30 \mathrm{~min}$ at $37^{\circ} \mathrm{C}$. Apoptosis was evaluated using a FACS Calibur flow cytometer (Becton Dickinson, Franklin Lakes, NJ, USA) with Annexin V-FITC and PI (KeyGEN Biotech, Nanjing, China) staining.

Western blot analysis. Cellular protein extracts were separated by electrophoresis on a 12 or $8 \%$ SDS-polyacrylamide gel and electrophoretically transferred onto a PDVF membrane (Millipore, Bedford, MA, USA). The membranes were blocked overnight with $5 \%$ non-fat dried milk and then incubated overnight at $4{ }^{\circ} \mathrm{C}$ with antibodies (Abs) directed against GAPDH [rabbit monoclonal (m)Ab; 1:1,500 dilution; cat no. 2118], PDK1 (rabbit mAb; 1:1,000 dilution; cat no. 13037), p-Akt (mouse mAb; 1:1,000 dilution; cat no. 4051), mTOR(p) (rabbit mAb; 1:1,000 dilution; cat no. 5536), p70S6K(p) (mouse Ab; 1:1,000 dilution; cat no. 9209), and 4E-BP1 (rabbit mAb; 1:1,000 dilution; cat no. 9456) from Cell Signaling Technology (Danvers, MA, USA) or TUSC4 (mouse Ab; 1:1,000 dilution; cat no. sc-376986), PI3K(p) [rabbit polyclonal(Ab); 1:1,000 dilution; cat no. sc-134986], caspase-3 (rabbit pAb; 1:1,000 dilution; cat no. sc-7148), and caspase-9 (mouse mAb; 1:2,000 dilution; cat no. sc-56073) from Santa Cruz Biotechnology (Santa Cruz, CA, USA). After washing with TBST, the membranes were incubated with horseradish peroxidase-linked goat anti-rabbit (1:1,000 dilution; cat no. A0208) and goat anti-mouse (1:1,000 dilution; cat no. A0216) IgG (heavy and light chain) secondary Abs (Beyotime Institute of Biotechnology). The proteins were visualized by ECL chemiluminescence using an integrated automatic chemiluminescent imaging and analysis system (Sage Creation Science, Beijing, China).

Statistical analyses. All experimental data are presented as the mean \pm standard error of the mean. Differences between samples were analyzed using the two-tailed Student's $t$-test. $\mathrm{P}<0.05$ was considered to indicate a statistically significant difference.

\section{Results}

Lentiviral transduction of TUSC4. Transduction efficiency was evaluated $72 \mathrm{~h}$ after TUSC4 transduction. eGFP was expressed in cells after lentiviral transduction at different multiplicities of infection (MOIs). The transduction efficiency (average proportion of GFP-expressing cells compared with the total cell count) was $>70 \%$ at an MOI of 10 . The protein expression levels were analyzed at $72 \mathrm{~h}$ post-transduction. TUSC4 expression was increased in the transduced cells compared with the negative control (NC) and mock cells (unloaded lentivirus) $(\mathrm{P}<0.05$, Fig. 1).

TUSC4 overexpression increases the sensitivity of HCT116 cells to 5-FU. To investigate the role of TUSC4 in 5-FU-induced cytotoxicity, TUSC4 was transduced into HCT116 cells. The $\mathrm{IC}_{50}$ of 5-FU was reduced in cells transduced with TUSC4 compared with in NC cells $(\mathrm{P}<0.05)$, indicating that overexpression of TUSC4 markedly increased the 5-FU sensitivity of HCT116 cells (Fig. 2A). Cell survival was assayed following TUSC4 transduction and treatment with $5 \mu \mathrm{M} 5$-FU for an additional 24,48 , and $72 \mathrm{~h}$, which 


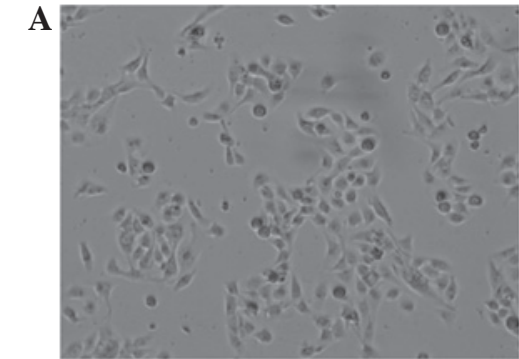

C

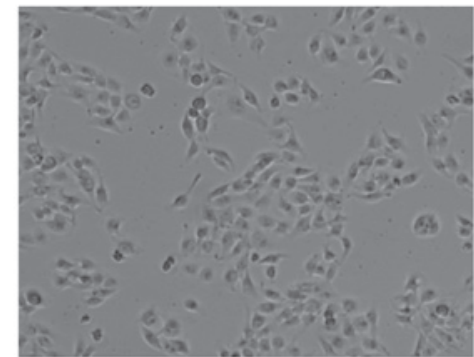

$\mathbf{E}$

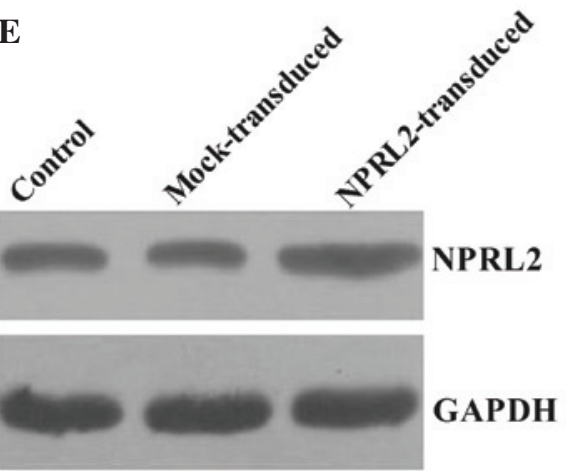

B

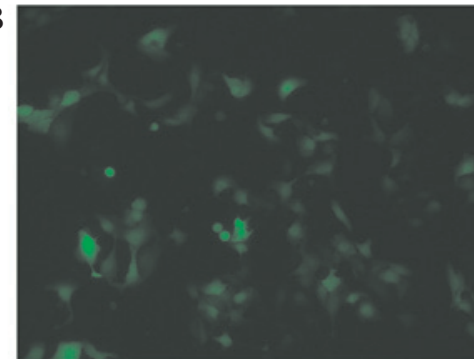

D

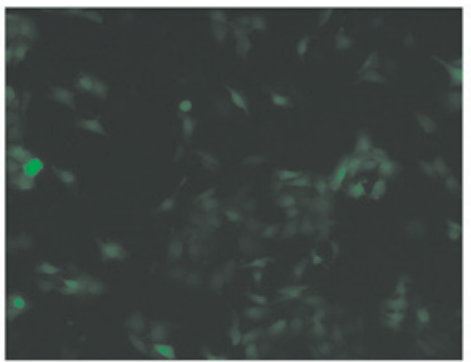

$\mathbf{F}$

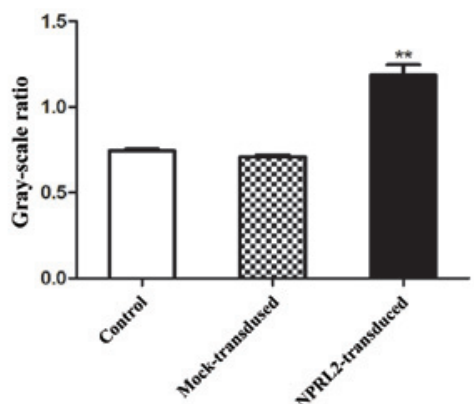

Figure 1. Monitoring the transduction efficiency of HCT116 cells by light and fluorescence microscopy. (A) HCT116 cells prior to mock-transduction, (B) mock-transduced HCT116 cells, (C) HCT116 cells prior to TUSC4-transduction (D) TUSC4-transduced HCT116 cells. The TUSC4 (NPRL2) protein expression levels were analyzed (E) by western blotting, (F) which was quantified by densitometry. ${ }^{* *} \mathrm{P}<0.01$ vs. control and mock-transduced cells.
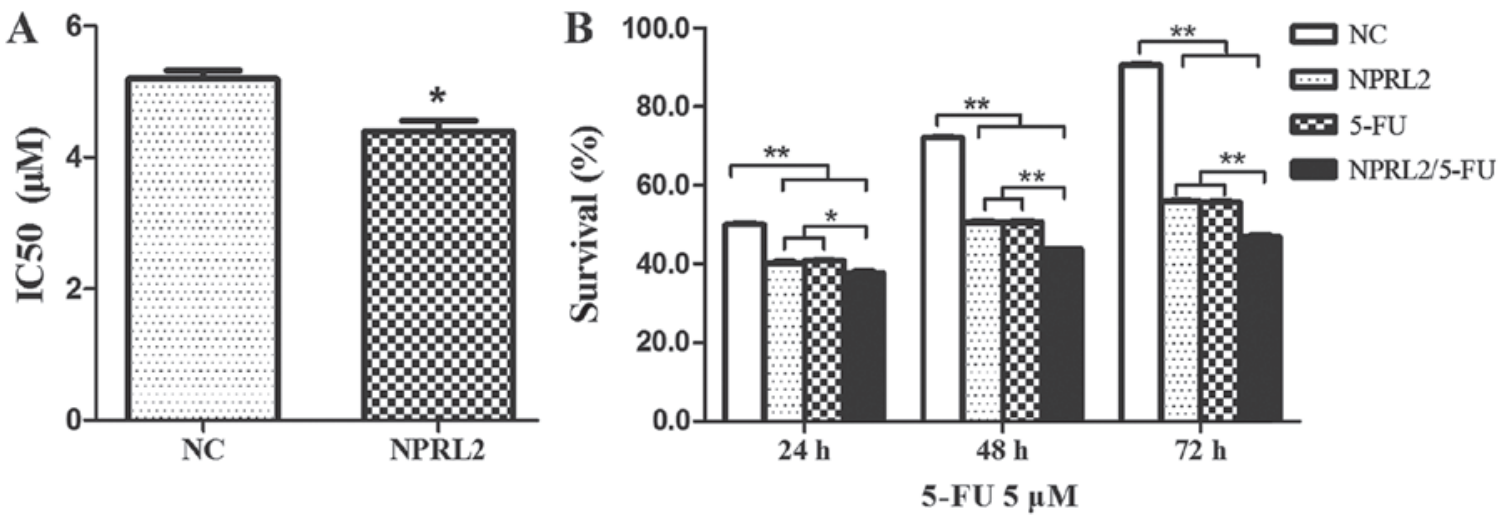

Figure 2. TUSC4 increases the 5-FU sensitivity of HCT116 cells. (A) The $\mathrm{IC}_{50}$ of 5-FU was reduced in cells transduced with TUSC4 compared with negative control (NC) cells. (B) Cell survival was assayed following TUSC4 transduction and treatment with $5 \mu \mathrm{M} 5$-FU for an additional 24, 48, and 72 h; " $\mathrm{P}<0.05$; ${ }^{* * *} \mathrm{P}<0.01$.

demonstrated that the effects of TUSC4 on 5-FU sensitivity were time dependent (Fig. 2B): Cell survival in 5-FU-treated TUSC4-transduced cells reduced over time $(\mathrm{P}<0.01)$.

TUSC4 overexpression increases 5-FU sensitivity by inhibiting cell growth. Cell survival assays demonstrated that a proportion of cells were was arrested in the G1 phase and that there was a reduction in the $\mathrm{S}$ phase population following TUSC4 transduction of HCT116 cells $(\mathrm{P}<0.05)$. In addition, 5-FU significantly inhibited the growth of TUSC4-transduced cells compared to $\mathrm{NC}$ cells and further promoted this effect ( $\mathrm{P}<0.01$; Fig. 3). 

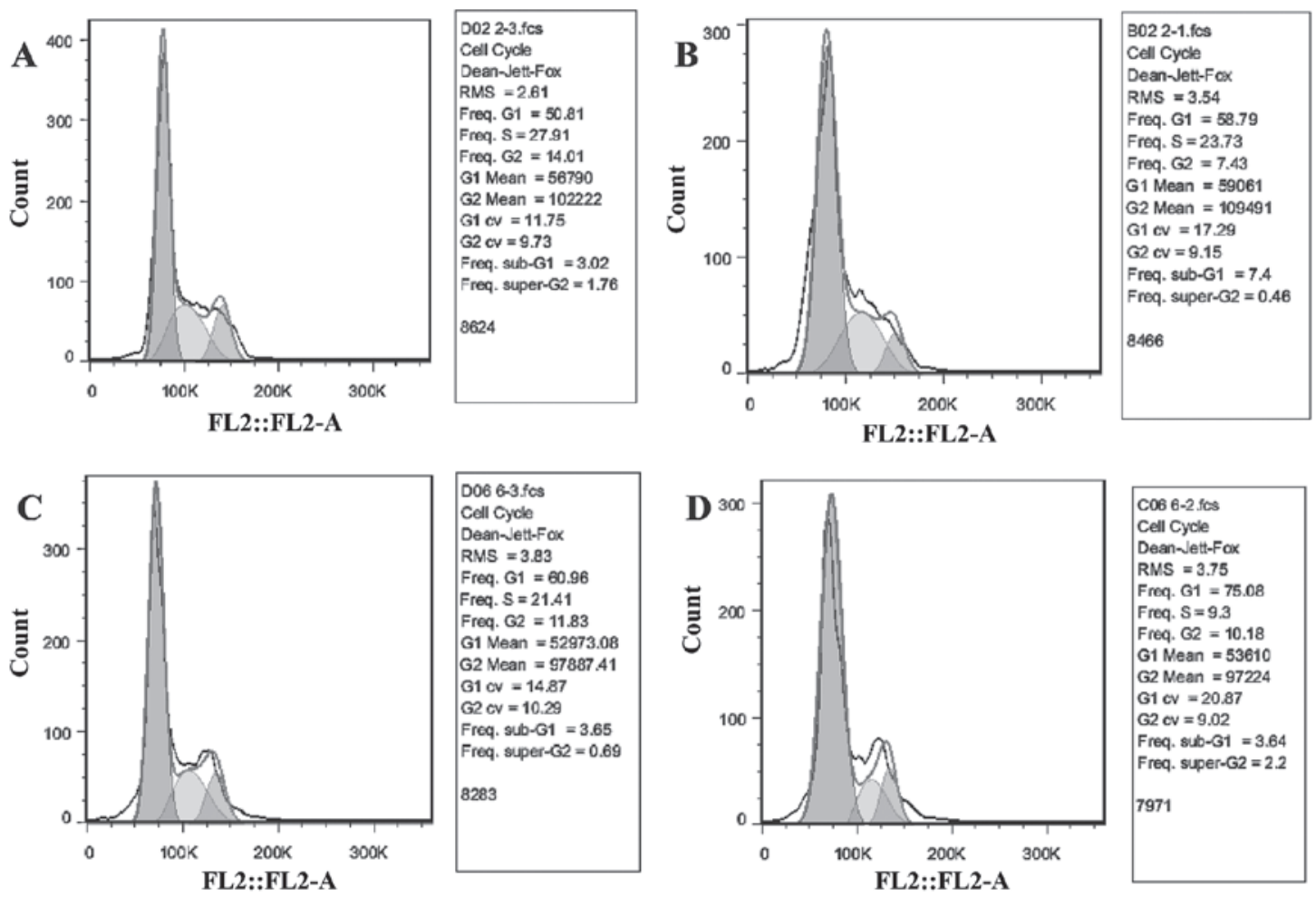

Figure 3. TUSC4 overexpression increases 5-FU sensitivity by inhibiting HCT116 cell growth. (A) Negative control (NC) cells; (B) TUSC4-transduced cells; (C) $48 \mathrm{~h}$ after HCT116 cells were treated with $5 \mu \mathrm{M} 5$-FU; (D) $48 \mathrm{~h}$ after TUSC4-transduced HCT116 cells were treated with $5 \mu \mathrm{M} 5$-FU.
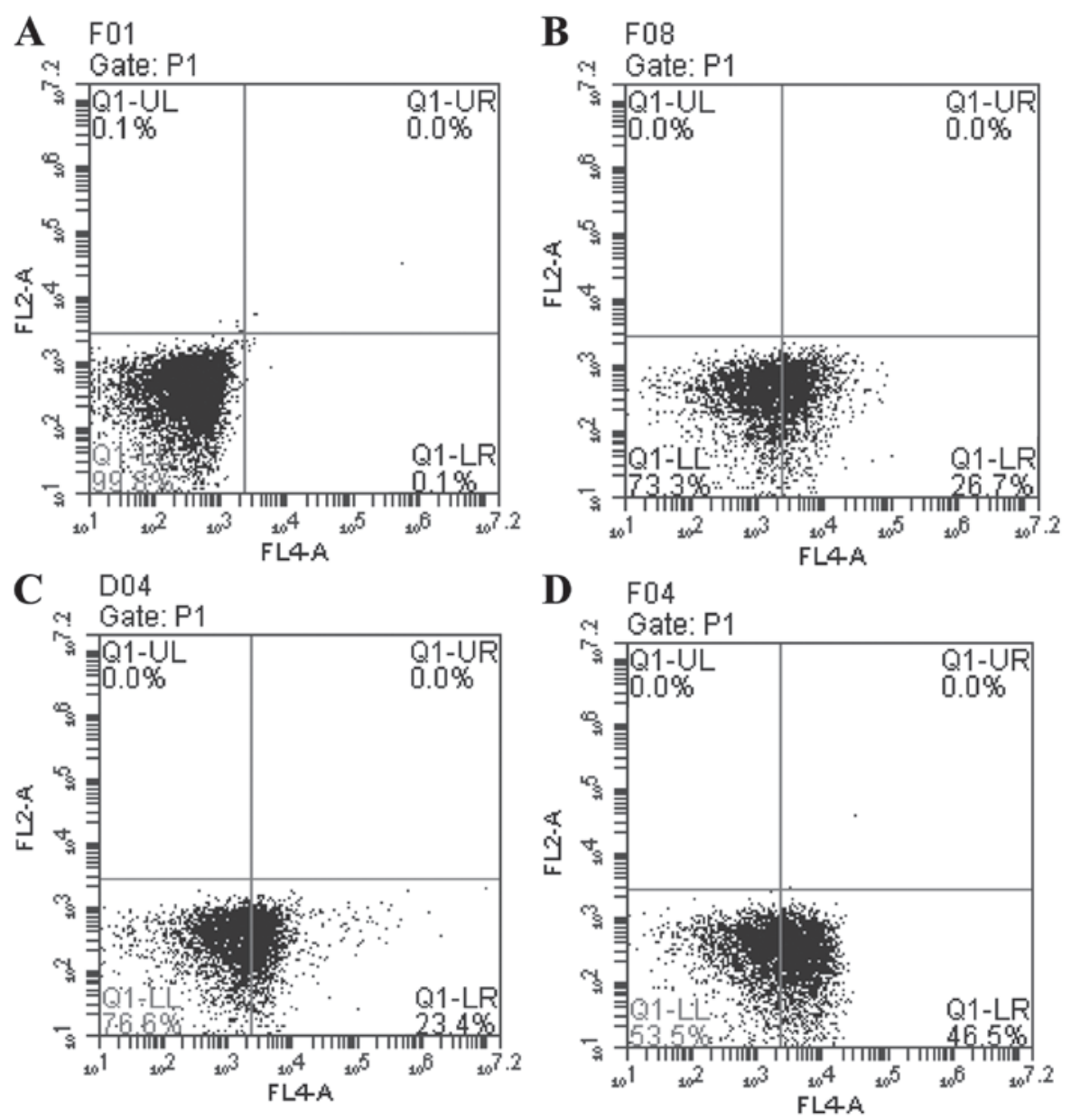

Figure 4. TUSC4 over-expression increases 5-FU sensitivity by promoting apoptosis in HCT116 cells. (A) NC cells; (B) TUSC4-transduced cells; (C) 48 h after HCT116 cells were treated with $5 \mu \mathrm{M}$ 5-FU; (D) $48 \mathrm{~h}$ after TUSC4-transduced HCT116 cells were treated with $5 \mu \mathrm{M}$ 5-FU. 


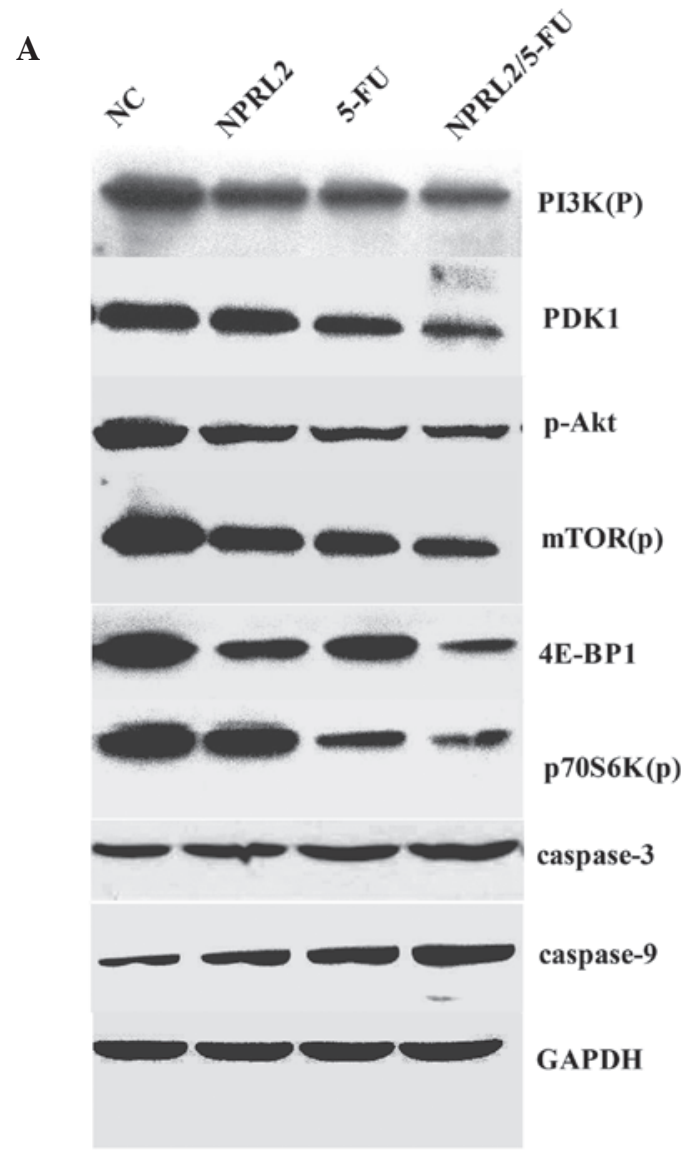

B

Figure 5. Western blot analysis of the protein levels of PI3K(p), PDK1, p-Akt, mTOR(p), p70S6K(p), 4E-BP1, caspase-3, caspase-9, and GAPDH. (A) The down-regulation of the expression of PDK1, 4E-BP1, phosphorylated PI3K, Akt, mTOR(p), and p70S6K(p) in cells overexpressing NPRL2 and treated with 5-FU compared with NC cells, NPRL2-transduced cells, and cells treated with $5 \mu \mathrm{M} 5$-FU. NPRL2 overexpression resulted in increased levels of caspase-3 and caspase-9 compared with NC cells. (B) In addition, combined NPRL2 over-expression and L-OHP treatment significantly upregulated apoptosis compared with either perturbation alone. Western blot analysis was performed using GAPDH as a loading control, ${ }^{* *} \mathrm{P}<0.01 \mathrm{vs.} \mathrm{NC,} \mathrm{NPRL2}$ and 5-FU.

TUSC4 overexpression increases 5-FU sensitivity by promoting apoptosis. Flow cytometry was performed to investigate the role of TUSC4 in 5-FU-induced apoptosis. The number of apoptotic cells was greater in TUSC4-transduced and 5-FU cells compared with NC cells $(\mathrm{P}<0.05)$. In addition, the combination of TUSC4 overexpression and 5-FU treatment promoted apoptosis more significantly than either perturbation alone $(\mathrm{P}<0.01)$ (Fig. 4).

TUSC4 promotes HCT116 cell sensitivity to 5-FU by inhibiting the PI3K/Akt/mTOR signaling pathway. The protein expression in the following 4 groups of cells was evaluated by western blot analysis: negative control cells, TUSC4-transduced cells, HCT116 cells treated with $5 \mu \mathrm{M} 5$-FU and cultured for $48 \mathrm{~h}$, and TUSC4-transduced HCT116 cells treated with $5 \mu \mathrm{M} 5$-FU and cultured for $48 \mathrm{~h}$. Overexpression of TUSC4 and 5-FU treatment downregulated PDK1, 4E-BP1, phosphorylated PI3K, Akt, mTOR, and p70S6K in HCT116 cells. In addition, the combination of TUSC4 overexpression and 5-FU treatment resulted in significantly greater downregulation of these genes compared to either perturbation alone $(\mathrm{P}<0.01)$. Furthermore, 5-FU upregulated caspase- 3 and caspase- 9 , promoting apoptosis in TUSC4 overexpressing cells compared with cells subjected to either perturbation alone or NC cells $(\mathrm{P}<0.01$; Fig. 5$)$.

\section{Discussion}

5-FU has been the first-choice chemotherapy drug for colorectal cancer for a number of years. Although the combination of 5-FU with other chemotherapeutic agents improves response rates and survival in breast and head and neck cancers, 5-FU has the greatest impact in the treatment of colorectal cancer (13). Nonetheless, the response rates for 5-FU-based chemotherapy as a first-line treatment for advanced colorectal cancer are only $\sim 10-15 \%$ (14). Additionally, the use of 5-FU as a colorectal cancer chemotherapeutic agent has been somewhat limited due to the toxicity, limited success and associated adverse side effects. Although the exact mechanism involved in the inactivation of TUSC4 in human cancers has not been determined, dysfunctional alterations in the TUSC4 gene and its products, including aberrant splicing transcripts and intragenic homozygous deletions, have been observed in various types of human cancer and cancer cell lines $(5,11)$. Recently, it was demonstrated that TUSC4 has tumor suppressing potential in vitro and in vivo, and TUSC4 may be involved in DNA mismatch repair, cell cycle checkpoint signaling, and the regulation of the apoptotic pathway $(9,11,15)$. Previous reports also demonstrated that NPRL2 is inactivated in various types of human cancer and cancer cell lines by aberrant splicing of its transcripts and/or intragenic homozygous deletions $(5,11)$. 
Additionally, exogenous NPRL2 expression in NPRL2-negative tumor cells activates the DNA damage pathway in lung cancer cells following NPRL2 treatment (12).

The present study demonstrated that TUSC4 over-expression increases 5-FU sensitivity in HCT116 cells. It was initially determined that the $\mathrm{IC}_{50}$ of $5-\mathrm{FU}$ was reduced in cells transduced with TUSC4 than in NC cells $(\mathrm{P}<0.05)$. The effects of TUSC4 on 5-FU sensitivity were determined to be time dependent. Following TUSC4 transduction in HCT116 cells, the cell cycle was arrested in G1 phase, and there was a reduction in cells in $\mathrm{S}$ phase $(\mathrm{P}<0.05)$. In addition, 5-FU significantly inhibited cell growth in TUSC4-transduced cells compared with $\mathrm{NC}$ cells $(\mathrm{P}<0.01)$. These data further confirm that TUSC4 overexpression increases 5-FU sensitivity by inhibiting cell growth. Flow cytometry analysis revealed an increase in apoptotic cells due to TUSC4 transduction and 5 -FU treatment compared to $\mathrm{NC}$ cells $(\mathrm{P}<0.05)$. Moreover, combined TUSC4 over-expression and 5-FU treatment promoted apoptosis more significantly than either perturbation alone. These findings indicate that there is a significant correlation between TUSC4 protein expression and 5-FU sensitivity in colorectal cancer cells, which is consistent with the hypothesis that TUSC4 serves a role in regulating the DNA-damage repair pathway and that inactivation of TUSC4 in tumor cells may promote drug resistance, which is likely to be due to interrupting DNA-damage repair and apoptotic signaling.

TUSC4 may enhance 5-FU sensitivity via additional mechanisms, which should be examined in further studies. In the present study, it was demonstrated that TUSC4 is a novel potential therapeutic molecule. Compared with traditional drugs, TUSC4 serves a more modulatory role. Combined TUSC4 overexpression and 5-FU treatment effectively downregulated the phosphorylation of phosphoinositide-3 kinase (PI3K), Akt, and mTOR in addition to the mTOR downstream target proteins phospho-p70S6K (Thr389) and 4E-BP1 (Thr37/46). The PI3K/Akt/mTOR signaling axis is critical for proliferation, apoptosis resistance, angiogenesis, and metastasis and is central to the development and maintenance of CRC (16). Previous studies have reported the potential for the PI3K/Akt/mTOR network to be therapeutically targeted at multiple molecular levels $(17,18)$. Activated PI3K generates a second messenger phosphatidylinositol(3-5)-triphosphate (PIP3); PIP3 then binds to and activates phosphoinositide-dependent kinase-1 (PDK1), which phosphorylates and activates Akt $(19,20)$. Akt activates several downstream targets, including mTOR. Deregulation of mTOR signaling occurs in several types of human tumor, including colon cancer $(16,20)$. mTOR resides in 2 distinct multiprotein complexes, mTORC1 and mTORC2 (21). mTORC1 directly phosphorylates ribosomal protein S6 kinase 1 (S6K1) and the eukaryotic translation initiation factor eIF4E-binding protein 1 (4EBP1), which are involved in protein translation $(22,23)$. Phospho-p70S6K is usually located in the cytoplasm, but it is often observed in the nucleus in tumors. Phospho-p70S6K stimulates ribosome rearrangement into active polysomes and increases the capacity of the translational events that are essential for the G1/S transition of the cell cycle (24). 4E-BP1 is considered a funneling factor through which transforming signals converge, channeling oncogenic proliferative signals regardless of the specific upstream oncogenic alteration (25). These findings indicate that TUSC4 overexpression enhances 5-FU sensitivity by downregulating the functions of the PI3K/Akt/mTOR network, leading to the inhibition of cell proliferation and G1 cell cycle arrest. Furthermore, 5-FU upregulates caspase-3 and caspase-9 to promote apoptosis in TUSC4 overexpressing cells compared with either perturbation alone and $\mathrm{NC}$ cells $(\mathrm{P}<0.01)$. In addition, the present study demonstrated that the TUSC4-mediated increase in 5-FU sensitivity that induces apoptosis in HCT116 cells is associated with significant activation of caspase-3 and caspase-9.

In summary, the present study demonstrates for the first time that the expression of endogenous TUSC4 significantly increased sensitivity to 5-FU in colorectal cancer cells. The results provide novel evidence and previously unrecognized effects of TUSC4, demonstrating that targeting TUSC4 in combination with the conventional colorectal cancer chemotherapeutic agent 5-FU may serve as an effective therapeutic strategy, resulting in the significant inhibition of colorectal cancer cell growth by downregulating the function of the PI3K/Akt/mTOR network. These mechanisms are likely active in other cancers and may be exploited for the development of novel cancer therapies.

\section{References}

1. Siegel R, Naishadham D and Jemal A: Cancer statistics, 2012. CA Cancer J Clin 62: 10-29, 2012.

2. Patel BB and Majumdar AP: Synergistic role of curcumin with current therapeutics in colorectal cancer: Minireview. Nutr Cancer 61: 842-6, 2009

3. Borralho PM, Moreira da Silva IB, Aranha MM, Albuquerque C and Nobre Leitao C: Inhibition of Fas expression by RNAi modulates 5-fluorouracilinduced apoptosis in HCT116 cells expressing wild-type p53. Biochim Biophys Acta 1772: 40-47, 2007.

4. Peters GJ, van Triest B, Backus HH, Kuiper CM, van der Wilt CL and Pinedo HM: Molecular downstream events and induction of thymidylate synthase in mutant and wild-type p53 colon cancer cell lines after treatment with 5-fluorouracil and the thymidylate synthase inhibitor raltitrexed. Eur J Cancer 36: 916-924, 2000.

5. Lerman MI and Minna JD: The 630-kb lung cancer homozygous deletion region on human chromosome 3 p21.3: Identification and evaluation of the resident candidate tumor suppressor genes. The International lung cancer chromosome $3 \mathrm{p} 21.3$ tumor suppressor gene consortium. Cancer Res 60: 6116-6133, 2000.

6. Wistuba II, Behrens C, Virmani AK, Mele G, Milchgrub S, Girard L, Fondon JW III, Garner HR, McKay B, Latif F, et al: High resolution chromosome $3 \mathrm{p}$ allelotyping of human lung cancer and preneoplastic/preinvasive bronchial epithelium reveals multiple, discontinuous sites of $3 p$ allele loss and three regions of frequent breakpoints. Cancer Res 60: 1949-1960, 2000.

7. Zabarovsky ER, Lerman MI and Minna JD: Tumor suppressor genes on chromosome $3 p$ involved in the pathogenesis of lung and other cancers. Oncogene 21: 6915-6935, 2002.

8. Ji L, Nishizaki M, Gao B, Burbee D, Kondo M, Kamibayashi C, Xu K, Yen N, Atkinson EN, Fang B, et al: Expression of several genes in the human chromosome 3 p21.3 homozygous deletion region by an adenovirus vector results in tumor suppressor activities in vitro and in vivo. Cancer Res 62: 2715-2720, 2002.

9. Schenk PW, Brok M, Boersma AW, Brandsma JA, Den Dulk H, Burger H, Stoter G, Brouwer J and Nooter K: Anticancer drug resistance induced by disruption of the Saccharomyces cerevisiae NPR2 gene: A novel component involved in cisplatin- and doxorubicin-provoked cell kill. Mol Pharmacol 64: 259-268, 2003.

10. Ueda K, Kawashima H, Ohtani S, Deng WG, Ravoori M, Bankson J, Gao B, Girard L, Minna JD and Roth JA: The 3p21.3 tumor suppressor NPRL2 plays an important role in cisplatin-induced resistance in human non-small-cell lung cancer cells. Cancer Res 66: 9682-9690, 2006. 
11. Li J, Wang F, Haraldson K, Protopopov A, Duh FM, Geil L, Kuzmin I, Minna JD, Stanbridge E, Braga E, et al: Functional characterization of the candidate tumor suppressor gene NPRL2/G21 located in 3p21.3C. Cancer Res 64: 6438-6443, 2004.

12. Jayachandran G, Ueda K, Wang B, Roth JA and Ji L: NPRL2 sensitizes human non-small cell lung cancer (NSCLC) cells to cisplatin treatment by regulating key components in the DNA repair pathway. PLoS One 8: e11994, 2010.

13. Efficacy of adjuvant fluorouracil and folinic acid in colon cancer. International multicentre pooled analysis of colon cancer trials (IMPACT) investigators. Lancet 345: 939-944, 1995.

14. Johnston PG and Kaye S: Capecitabine: A novel agent for the treatment of solid tumors. Anticancer Drugs 12: 639-646, 2001.

15. Zhou BB and Elledge SJ: The DNA damage response: Putting checkpoints in perspective. Nature 408: 433-439, 2000.

16. Johnson SM, Gulhati P, Rampy BA, Han Y, Rychahou PG, Doan HQ, Weiss HL and Evers BM: Novel expression patterns of PI3K/Akt/mTOR signaling pathway components in colorectal cancer. J Am Coll Surg 210: 767-776, 2010.

17. Maira SM, Voliva C and Garcia-Echeverria C: Class IA phosphatidylinositol 3-kinase: From their biologic implication in human cancers to drug discovery. Exp Opin Ther Targets 12: 223-238, 2008.

18. Ekstrand AI, Jönsson M, Lindblom A, Borg A and Nilbert M Frequent alterations of the PI3K/AKT/mTOR pathways in hereditary nonpolyposis colorectal cancer. Fam Cancer 9: $125-129,2010$
19. Samuels $\mathrm{Y}$ and Ericson K: Oncogenic PI3K and its role in cancer. Curr Opin Oncol 18: 77-82, 2006.

20. Vignot S, Faivre S, Aguirre D and Raymond E: mTOR-targeted therapy of cancer with rapamycin derivatives. Ann Oncol 16: $525-537,2005$

21. Guertin DA and Sabatini DM: Defining the role of mTOR in cancer. Cancer Cell 12: 9-22, 2007.

22. Tabernero J, Rojo F, Calvo E, Burris H, Judson I, Hazell K, Martinelli E, Ramon y Cajal S, Jones S, Vidal L, et al: Doseand-and schedule-dependent inhibition of the mammalian target of rapamycin pathway with everolimus: A phase I tumor pharmacodynamic study in patients with advanced solid tumors. J Clin Oncol 26: 1603-1610, 2008.

23. Proud CG: mTORC1 signalling and mRNA translation. Biochem Soc Trans 37: 227-231, 2009.

24. Xu G, Zhang W, Bertram P, Zheng XF and McLeod H: Pharmacogenomic profiling of the PI3K/PTEN-AKT-mTOR pathway in common human tumors. Int J Oncol 24: 893-900, 2004.

25. Armegnol G, Rojo F, Castellvi Ĺ, Iglesias C, Cuatrecasas M, Pons B, Baselga J and Ramón y Cajal S: 4E-binding protein 1: A key molecular 'funnel factor' in human cancer with clinical implications. Cancer Res 67: 7551-7555, 2007. 